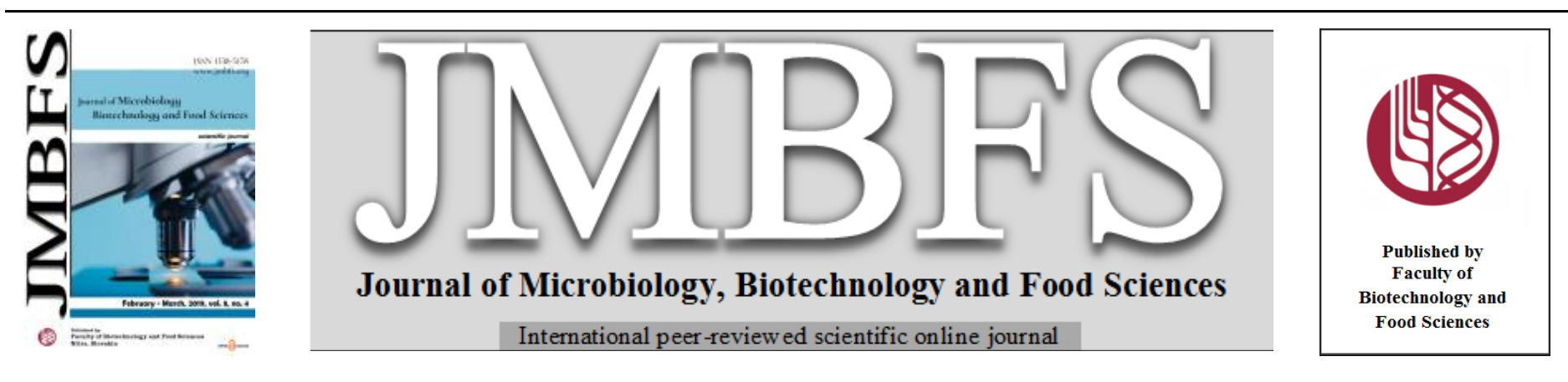

\title{
NEW APPROACH FOR FAST SCREENING OF LACTIC ACID BACTERIA FOR VEGETABLE FERMENTATION
}

\author{
Inna Garmasheva*, Olga Vasyliuk, Nadezhda Kovalenko, and Ljubov Oleschenko
}

Address(es): Dr. Inna Garmasheva,

Zabolotny Institute of Microbiology and Virology National Academy of Science of Ukraine, Department of Physiology of Industrial Microorganisms, 154 Zabolotnogo str, Kyiv 03143, Ukraine, +38 0445262329.

*Corresponding author: garmasheva.il@gmail.com

doi: 10.15414/jmbfs.2019.8.4.1066-1071

\section{ARTICLE INFO}

Received 24. 4. 2018

Revised 12.11. 2018

Accepted 13. 11. 2018

Published 1. 2. 2019

Regular article

OPEN $\partial_{\text {ACCESS }}$

\begin{abstract}
The main goal of this research was to select a Lactobacillus plantarum strains which could be used as starters for fermented vegetables production. A total of $109 \mathrm{~L}$. plantarum strains were screened based on their ability to drop the $\mathrm{pH}$ value in pasteurized vegetable-based media (without and with $\mathrm{NaCl}$ ) after $8 \mathrm{~h}$ incubation. The effect of $\mathrm{NaCl}$ on the level of acidification after $8 \mathrm{~h}$ incubation was more dependent not on its concentration but on the vegetable-based medium and L. plantarum strain. Four L. plantarum strains were selected and used for lab-scale fermentation of cabbage and cucumbers, this resulted in an accelerated acidification of the vegetables in comparison with the spontaneous fermentation. Strong positive correlations between the differences of initial $\mathrm{pH}$ value and the values reached after fermentation during $8 \mathrm{~h}(\Delta \mathrm{pH})$ on vegetable-based media and vegetable fermentations, suggests that the screening process in vegetable-based media after $8 \mathrm{~h}$ incubation could be effective. Four $L$. plantarum strains, selected in this study, can be used as starters for fermented vegetables.
\end{abstract}

Keywords: fermentation, vegetables, Lactobacillus, biotechnology, fermented foods

\section{INTRODUCTION}

Fermented vegetables have been produced worldwide for thousands of years mainly for preservation purposes (Gorny et al., 2006; Das et al., 2016). Some traditional methods are still used to manufacture naturally fermented vegetables from raw vegetables. A rapid decrease in $\mathrm{pH}$ at the beginning of fermentation is of great importance for the quality of the end product (Viander et al., 2003). But spontaneous fermentation generally needs 4 to 6 days before the pH stabilizes, and depends on the competitive activities of the indigenous microbiota, mainly lactic acid bacteria (LAB). Lactobacillus plantarum is one of the species of LAB most frequently found in the spontaneous fermentation of different vegetables, such as cucumbers (Tamminen et al., 2004), table olives (Harris, 1998), kimch (Kim and Chun, 2005) and cabbages (Karovičová and Kohajdová, 2003; Soltan Dallal et al., 2017). The frequent appearance of $L$. plantarum strains during vegetable fermentations makes this species a promising candidate for starter culture trials. A large number of LAB starters are routinely used in dairy and meat industry, but only a few cultures designed for vegetable fermentations (Montet et al., 2006). There are some differences between processes in producing dairy products and fermented vegetables production. The main difference is the inability to inactivate the endogenous microbiota of raw vegetables by a heat treatment without causing drastic changes in their texture. Nevertheless, an increasing number of studies concerning the use of starter culture for fermented vegetables production is published (Lee $\boldsymbol{e t}$ al., 2018; Joyce et al., 2018). The most common approach for screening of LAB strains for vegetable fermentation is the evaluation of ability of growth and acidification in sterilized vegetable juices (Gardner et al., 2001; Di Cagno et al., 2008). But the unified evaluation criteria have not been developed. At the same time, there is a criterion for fast evaluation of acidification ability for dairy starters according to the $\mathrm{pH}$ value reached in sterile, reconstituted (10\% w/v) skimmed milk after 6-8 h incubation (Cogan et al., 1997; Roushdy, 1999). In our opinion, the $\mathrm{pH}$ value of pasteurized vegetable-based medium after $8 \mathrm{~h}$ incubation might be a good criterion for selection of LAB strains suitable for producing fermented vegetables.

Fermented vegetables have played a significant role in the diet of the population of Ukraine for many years. However, at the moment, there is a lack of commercial bacterial cultures suitable for fermentation of vegetables in Ukraine. Moreover, studies on screening and characterization of Lactobacillus plantarum strains with potential use as starter cultures for vegetables fermentation in Ukraine are limited. In our previous works a total of 109 strains of $L$. plantarum were isolated from Ukrainian dairy products and fermented vegetables and these were studied for various biochemical and functional properties (Vasyliuk $\boldsymbol{e t}$ al. 2014a, b). So, starter cultures with desirable properties are of particular importance for the production of fermented vegetable products with functional properties (Soltan Dallal et al., 2017; Behera et al., 2018)

Hence, the aim of this work was to assess the potential of L. plantarum strains, isolated from Ukrainian fermented products, for fermented vegetable production. For this purpose, the suitability of a simple approach, based on the $\mathrm{pH}$ value of pasteurized vegetable-based media after $8 \mathrm{~h}$ incubation, as a criterion for possible vegetable starter cultures, was assessed. Selected $L$. plantarum strains were successfully applied on lab-scale for fermentation of cabbage and cucumbers, following a traditional production method.

\section{MATERIAL AND METHODS}

\section{Bacterial strains}

One hundred and nine L. plantarum strains isolated from various homemade fermented foods and sampled in different regions in Ukraine were used (Vasyliuk et al., 2014). All strains were maintained in MRS broth, with the addition of $30 \%(\mathrm{w} / \mathrm{v})$ glycerol at $-50{ }^{\circ} \mathrm{C}$ in the $\mathrm{LAB}$ Culture Collection of the Department of Physiology of Industrial Microorganisms, Zabolotny Institute of Microbiology and Virology NAS, Kiev, Ukraine. The cultures were activated by two successive transfers in the MRS broth before use.

\section{Initial screening}

For the initial screening of the strains, a vegetable-based media were produced. Fresh cabbage and cucumbers were washed, cut and separately homogenized in sterile distilled water, at ratios of 1:1 (vegetable : water). $\mathrm{NaCl}$ was added at the concentration of $2.5 \%(\mathrm{w} / \mathrm{v})$ to cabbage-based medium and $6.0 \%$ to cucumberbased medium. Media without $\mathrm{NaCl}$ are also used. All media were pasteurized at $80{ }^{\circ} \mathrm{C}$ for $20 \mathrm{~min}$, cooled and immediately inoculated with $1 \%(\mathrm{v} / \mathrm{v})$ of an overnight culture of tested strains. Media were incubated for $8 \mathrm{~h}$ at $30{ }^{\circ} \mathrm{C}$ and acidification was determined by measurement of the $\mathrm{pH}$ of samples and expressed as the difference in $\mathrm{pH}$ between the initial $\mathrm{pH}$ value and the value reached after $8 \mathrm{~h}(\Delta \mathrm{pH})$. Non-inoculated media were used as a control (Table 1). 
Table 1 The $\mathrm{pH}$ values of vegetable-based media

\begin{tabular}{|c|c|c|c|c|}
\hline \multirow{2}{*}{ Medium } & \multicolumn{2}{|c|}{ initial pH value } & \multicolumn{2}{|c|}{$\begin{array}{c}\mathrm{pH} \text { value after } 8 \mathrm{~h} \\
\left(\text { fermentation at } 30^{\circ} \mathrm{C} \text { ) }\right.\end{array}$} \\
\hline & $\begin{array}{c}\text { non- } \\
\text { pasterized }\end{array}$ & pasterized & $\begin{array}{c}\text { non- } \\
\text { pasterized }\end{array}$ & pasterized \\
\hline $\begin{array}{l}\text { Cabbage-based } \\
\text { medium }\end{array}$ & $5.66 \pm 0.31$ & $5.68 \pm 0.28$ & $5.41 \pm 0.28$ & $5.65 \pm 0.32$ \\
\hline $\begin{array}{l}\text { Cucumber-based } \\
\text { medium }\end{array}$ & $5.72 \pm 0.22$ & $5.62 \pm 0.31$ & $5.46 \pm 0.32$ & $5.56 \pm 0.30$ \\
\hline
\end{tabular}

\section{Vegetables fermentation}

Fresh cabbage was shredded, mixed with $\mathrm{NaCl}(2.5 \% \mathrm{w} / \mathrm{w})$ and placed in $500 \mathrm{~m}$ glass jars $(500 \mathrm{~g}$ of cabbage in each jar). Fresh cucumbers were washed and placed in 11 glass jars and $6.0 \% \mathrm{NaCl}$ solution $(\mathrm{w} / \mathrm{v})$ was added in each jar (520 $\pm 5 \mathrm{~g}$ of cucumbers with $460 \pm 5 \mathrm{ml}$ of brine). Jars with salted vegetables were inoculated with $1 \%(\mathrm{v} / \mathrm{v})$ of an overnight culture of $L$. plantarum strain to achieve an initial level of $10^{7} \mathrm{cfu} \mathrm{g}^{-1}$. The vegetables were mixed with inocula and packed tightly. Salted but not inoculated vegetables were used as a control, allowing a spontaneous fermentation. The fermentation was initiated at $30^{\circ} \mathrm{C}$ for 2 days, after which the jars were stored at $10{ }^{\circ} \mathrm{C}$ for the remainder of the fermentation. The fermentation process was performed in duplicate.

Brine and vegetable particles were sampled at specific time points throughout the fermentations, namely at day $0,1,2,3,14,21,28$, and after 3 months. Al samples were immediately analyzed for acidification and total lactic acid bacteria (LAB) count.

\section{Statistical analysis}

A one-way analysis of variance (ANOVA), multiple mean comparisons using LSD test (LSD - little significant difference), cluster analysis and graphing procedures were carried out with the Statistika 7.0 software (Systat Inc., USA) Results from two independent assays were averaged. Statistical significance was defined as $P<0.05$.

\section{RESULTS AND DISCUSSION}

\section{Growth of $L$. plantarum strains in vegetable-based media}

At the first stage, $109 \mathrm{~L}$. plantarum strains were screened based on the ability to grow in vegetable-based media. After $8 \mathrm{~h}$ of fermentation at $30^{\circ} \mathrm{C}$, the values of $\Delta \mathrm{pH}$ (the difference in $\mathrm{pH}$ between the initial $\mathrm{pH}$ value and the value reached after $8 \mathrm{~h}$ ) ranged from $0.125 \pm 0.02$ to $2.33 \pm 0.01$, depending on the medium and strain. A hierarchical cluster analysis was performed and grouping of $L$. plantarum strains on the base of $\Delta \mathrm{pH}$ values was mostly related to the differences of $\Delta \mathrm{pH}$ values on medium without $\mathrm{NaCl}$ and corresponding medium with $\mathrm{NaCl}$ and was unrelated with source of the strains (Fig. 1). For $10 \%$ strains, $\mathrm{NaCl}$ addition did not affect the $\Delta \mathrm{pH}$ values in both vegetable-based media (clusters I and III), whereas for $33 \%$ strains the $\mathrm{pH}$ values were lower than in the corresponding media without salt (clusters IV and VII). For other strains, the effect of $\mathrm{NaCl}$ on $\Delta \mathrm{pH}$ values was different depending on the vegetable-based medium. For $42.2 \%$ strains in cabbage-based medium with $2.5 \% \mathrm{NaCl}$ the $\Delta \mathrm{pH}$ values were lower, but in cucumber-based medium with $6 \% \mathrm{NaCl}$ the $\Delta \mathrm{pH}$ values were the same, compared with corresponding media without salt (clusters II and VI). For $11 \%$ strains (cluster VIII), the $\Delta \mathrm{pH}$ values for at least one of the vegetable-based media with salt (mainly cucumber-based with $6 \% \mathrm{NaCl}$ ) were higher in comparison with the corresponding medium without salt, whereas in the other medium the $\Delta \mathrm{pH}$ values did not change or were lower. So, an interesting observation made during this study was that the effect of $\mathrm{NaCl}$ on the level of acidification after $8 \mathrm{~h}$ incubation was more dependent not on its concentration but on the vegetable-based medium and $L$. plantarum strain. For many $L$. plantarum strains in cabbage-based medium with low $\mathrm{NaCl}$ concentration $(2.5 \%)$ the $\Delta \mathrm{pH}$ values were lower but in cucumber-based medium with high $\mathrm{NaCl}$ concentration $(6.0 \%)$, the $\Delta \mathrm{pH}$ values were higher, compared to corresponding $\mathrm{NaCl}$-free media.

\section{Vegetable fermentation}

Starter cultures with desirable functional properties, apart from the technological aspect, are of particular interest in terms of producing fermented vegetables with improved quality and usefulness for people health (Tamang et al., 2009; Rezac et al., 2018; Behera et al., 2018). Thus, in addition to acidification capacity in vegetable-based media, we took into account the functional properties of $L$. plantarum strains (Vasyliuk et al., 2014a,b; Livinska et al., 2016). So, four $L$ plantarum strains, namely L. plantarum 47SM (isolated from soured cream), 1047K, 952K (isolated from fermented cabbage) and 691T (isolated from cottage cheese) were selected to study their suitability as starter cultures for fermented cabbage and cucumbers.

The $\mathrm{pH}$ of the inoculated by $L$. plantarum strains cabbage was significantly $(P<0.05)$ lower than the control (Fig. 2a), whereas there were no significant differences in $\mathrm{pH}$ decreases between spontaneous fermented and inoculated cucumbers after $24 \mathrm{~h}$ (Fig. 2c). After a $48 \mathrm{~h}$ fermentation process, the $\mathrm{pH}$ values of inoculated cabbage samples decreased to a level below than 3.4. At the same time, the values of $\mathrm{pH}$ for cucumbers fermented with L. plantarum strains were in the range from 3.56-3.74. After $48 \mathrm{~h}$ incubation, the values of $\mathrm{pH}$ of spontaneously fermented vegetables were higher than those with $L$. plantarum strains.

The LAB growth dynamics significantly differed during fermentation of cabbage and cucumbers similarly to the difference in medium acidification dynamics. The counts of presumptive LAB were significantly higher after $24 \mathrm{~h}$ of incubation in the inoculated cabbage in contrast to control cabbage fermented spontaneously (Fig. 2b). However, there was no difference $(\mathrm{P}>0.05)$ in presumptive LAB counts between control and L. plantarum-inoculated cabbage after $48 \mathrm{~h}$. After $24 \mathrm{~h}$ fermentation, $\mathrm{LAB}$ cell count was not differed between control and inoculated cucumbers. The increase of presumptive LAB in cucumbers inoculated with $L$. plantarum $47 \mathrm{SM}$ occurred after $48 \mathrm{~h}$ becoming significantly higher compared to spontaneously fermented cucumbers (Fig. 2d). Overall, there were no significant differences in presumptive $\mathrm{LAB}$ counts and $\mathrm{pH}$ values between spontaneous fermented and inoculated vegetables under refrigeration storage during 3 months. 
$\triangle \mathbf{p H}$

without $\mathrm{NaCl} 6,6 \% \mathrm{NaCl}$ Source of isolatio

$0 \pm 0.170$ fermented apple $\left.\begin{array}{lllll}1.725+0.015 & 1.675+0.025 & 1.73+0.00 & \text { fermented cabbage }\end{array}\right]$ $\left.\begin{array}{lllll}1.49 \pm 0.01 & 1.35 \pm 0.09 & 1.175 \pm 0.025 & 1.07 \pm 0.02 & \text { fermented cabbage }\end{array}\right\}, \Delta \mathrm{pHcab}>\Delta \mathrm{pHcuc}$

$\begin{array}{lllll}1.98 \pm 0.03 & 1.815 \pm 0.035 & 1.63 \pm 0.21 & 1.515 \pm 0.015 & \text { fermented cabbage }\end{array}$ $\begin{array}{lllll}2.02 \pm 0.01 & 1.645 \pm 0.095 & 1.71 \pm 0.14 & 1.515 \pm 0.015 & \text { soured cream }\end{array}$ $\begin{array}{lllll}2.195 \pm 0.005 & 1.85 \pm 0.05 & 1.75 \pm 0.25 & 1.56 \pm 0.03 & \text { soured cream }\end{array}$ $\begin{array}{lllll}2.175 \pm 0.035 & 1.705 \pm 0.015 & 1.83 \pm 0.12 & 1.565 \pm 0.095 & \text { soured cream }\end{array}$ $\begin{array}{lllll}2.07 \pm 0.02 & 1.35 \pm 0.03 & 1.07 \pm 0.12 & 1.24 \pm 0.11 & \text { cottage cheese }\end{array}$ $\begin{array}{lllll}1.865 \pm 0.165 & 1.535 \pm 0.025 & 1.53 \pm 0.08 & 1.44 \pm 0.05 & \text { cottage cheese }\end{array}$ $\begin{array}{lllll}2.125 \pm 0.145 & 0.99 \pm 0.22 & 1.33 \pm 0.11 & 1.62 \pm 0.04 \quad \text { cottage cheese }\end{array}$ $\begin{array}{lllll}2.155 \pm 0.015 & 1.605 \pm 0.015 & 1.78 \pm 0.18 & 1.55 \pm 0.14 & \text { bryndza }\end{array}$ $\begin{array}{lllll}2.13 \pm 0.04 & 1.615 \pm 0.125 & 1.705 \pm 0.005 & 1.53 \pm 0.07 & \text { bryndza }\end{array}$

$\begin{array}{lllll}2.08 \pm 0.02 & 1.77 \pm 0.05 & 1.645 \pm 0.245 & 1.555 \pm 0.035 & \text { fermented cabbage }\end{array}$

$\begin{array}{lllll}2.04 \pm 0.00 & 1.72 \pm 0.04 & 1.73 \pm 0.23 & 1.65 \pm 0.04 & \text { fermented cabbage }\end{array}$ $\begin{array}{lllll}1.91 \pm 0.01 & 1.155 \pm 0.115 & 1.20 \pm 0.04 & 1.31 \pm 0.27 & \text { fermented cabbage }\end{array}$ $\begin{array}{lllll}2.15 \pm 0.03 & 1.61 \pm 0.13 & 1.735 \pm 0.035 & 1.61 \pm 0.03 & \text { fermented cabbage }\end{array}$ $\begin{array}{lllll}1.975 \pm 0.105 & 0.885 \pm 0.015 & 1.16 \pm 0.28 & 1.045 \pm 0.235 & \text { fermented cabbage }\end{array}$ $\begin{array}{lllll}2.14 \pm 0.01 & 1.375 \pm 0.075 & 1.485 \pm 0.195 & 1.36 \pm 0.18 & \text { fermented cabbage }\end{array}$ $\begin{array}{llll}1.865 \pm 0.385 & 1.375 \pm 0.025 & 1.70 \pm 0.26 & 1.59 \pm 0.05\end{array} \quad$ fermented cabbage

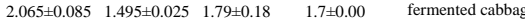
$\begin{array}{llll}1.995 \pm 0.155 & 1.255 \pm 0.115 & 1.695 \pm 0.285 & 1.45 \pm 0.03\end{array} \quad$ fermented cabbage $\begin{array}{lllll}2.065 \pm 0.025 & 1.41 \pm 0.08 & 1.865 \pm 0.085 & 1.59 \pm 0.05 & \text { fermented cabbage }\end{array}$ $\begin{array}{lllll}2.025 \pm 0.015 & 1.515 \pm 0.005 & 1.735 \pm 0.185 & 1.54 \pm 0.17 \quad \text { cottage cheese }\end{array}$ $\begin{array}{lllll}1.715 \pm 0.045 & 1.09 \pm 0.06 & 1.42 \pm 0.32 & 1.385 \pm 0.005 & \text { cottage cheese }\end{array}$ $\begin{array}{lllll}1.77 \pm 0.19 & 1.22 \pm 0.12 & 1.68 \pm 0.08 & 1.375 \pm 0.085 & \text { bryndza }\end{array}$ $\begin{array}{lllll}2.065 \pm 0.005 & 1.855 \pm 0.015 & 1.06 \pm 0.21 & 1.215 \pm 0.025 & \text { bryndza }\end{array}$ ] $\mathrm{pHcab}>\Delta \mathrm{pHcuc}$ $2.10 \pm 0.06 \quad 1.925+0.035 \quad 1.025+0.125 \quad 0.775+0.065$ fermented cabbage $\quad \Delta \mathrm{pHcab} 2.5 \% \mathrm{NaCl}>\Delta \mathrm{pHcuc} 6.0 \% \mathrm{NaCl} \quad \Delta \mathrm{pHcab}=\Delta \mathrm{pHcab} 2.5 \% \mathrm{NaCl}$ $\begin{array}{llll}1.975 \pm 0.0035 & 1.82 \pm 0.05 & 1.76 \pm 0.23 & 1.475 \pm 0.025\end{array}$ fermented cabbage $\begin{array}{lllll}1.26 \pm 0.36 & 1.45 \pm 0.02 & 1.69 \pm 0.13 & 1.52 \pm 0.11 & \text { fermented cabbage }\end{array}$ $\begin{array}{lllll}1.46 \pm 0.12 & 1.115 \pm 0.005 & 0.475 \pm 0.055 & 0.125 \pm 0.015 & \text { soured cream }\end{array}$ $\begin{array}{lllll}2.20 \pm 0.05 & 1.84 \pm 0.02 & 1.475 \pm 0.025 & 1.255 \pm 0.005 & \text { soured cream }\end{array}$ $2.195 \pm 0.025 \quad 1.22 \pm 0.02 \quad 1.18 \pm 0.02 \quad 0.705 \pm 0.005$ fermented cow's $m$ $\begin{array}{lllll}2.13 \pm 0.03 & 1.46 \pm 0.03 & 1.639 \pm 0.039 & 0.93 \pm 0.05 & \text { cottage cheese }\end{array}$ $\begin{array}{lllll}1.625 \pm 0.225 & 1.125 \pm 0.065 & 1.27 \pm 0.10 & 0.68 \pm 0.05 & \text { cottage cheese }\end{array}$ $\begin{array}{lllll}2.22 \pm 0.01 & 1.13 \pm 0.07 & 1.465 \pm 0.025 & 1.165 \pm 0.045 & \text { soured cream }\end{array}$ $\begin{array}{lllll}2.155 \pm 0.015 & 1.43 \pm 0.13 & 1.33 \pm 0.02 & 1.14 \pm 0.04 & \text { cottage cheese }\end{array}$ $\begin{array}{lllll}2.08 \pm 0.01 & 0.74 \pm 0.05 & 1.695 \pm .0235 & 0.84 \pm 0.09 & \text { fermented cabbage }\end{array}$

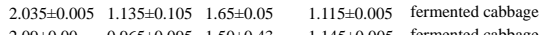
$\begin{array}{lllll}2.09 \pm 0.00 & 0.965 \pm 0.095 & 1.50 \pm 0.43 & 1.145 \pm 0.005 & \text { fermented cabbage } \\ 2.0100 .04 & 0.890 .10 & 1.39 & \end{array}$ $\begin{array}{lllll}2.01 \pm 0.04 & 0.89 \pm 0.10 & 1.39 \pm 0.03 & 0.705 \pm 0.005 & \text { fermented cabbage }\end{array}$

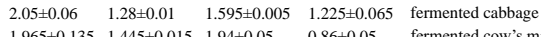

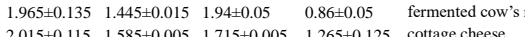
$\begin{array}{lllll}2.015 \pm 0.115 & 1.585 \pm 0.005 & 1.715 \pm 0.005 & 1.265 \pm 0.125 & \text { cottage cheese }\end{array}$ $\begin{array}{lllll}1.745 \pm 0.105 & 0.98 \pm 0.04 & 1.65 \pm 0.07 & 0.405 \pm 0.095 & \text { cottage cheese }\end{array}$ $\begin{array}{llll}1.885 \pm 0.055 & 1.565 \pm 0.015 & 1.65 \pm 0.23 & 0.74 \pm 0.09\end{array} \quad$ soured cream $\begin{array}{lllll}2.005 \pm 0.035 & 1.215 \pm 0.085 & 1.725 \pm 0.155 & 0.98 \pm 0.14 & \text { fermented cabbage }\end{array}$ $\begin{array}{lllll}1.94 \pm 0.06 & 1.355 \pm 0.22 & 1.905 \pm 0.18 & 1.435 \pm 0.025 & \text { fermented cabbage }\end{array}$ $\begin{array}{lllll}1.85 \pm 0.05 & 0.985 \pm 0.205 & 1.945 \pm 0.005 & 1.005 \pm 0.285 & \text { fermented cabbage }\end{array}$ $\begin{array}{lllll}2.095 \pm 0.125 & 1.30 \pm 0.05 & 1.89 \pm 0.18 & 1.405 \pm 0.055 & \text { fermented cabbage }\end{array}$ $\begin{array}{lllll}1.815 \pm 0.005 & 0.98 \pm 0.04 & 1.815 \pm 0.025 & 1.075 \pm 0.095 & \text { fermented cabbage } \\ 1.09 \pm 0.04 & 135 \pm 0.21 & 1.85 \pm 0.07 & 1.065 \pm 0.015 & \text { cottage cheese }\end{array}$ $\begin{array}{lllll}2.09 \pm 0.04 & 1.35 \pm 0.21 & 1.85 \pm 0.07 & 1.065 \pm 0.015 & \text { cottage cheese }\end{array}$

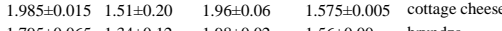

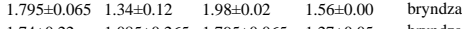
$\begin{array}{lllll}1.74 \pm 0.22 & 1.085 \pm 0.265 & 1.795 \pm 0.065 & 1.27 \pm 0.05 & \text { bryndza } \\ 1.75250 .015 & 1.07 \pm 0.0 & 1.54 \pm 0.07 & 0.095 \pm 0.055 & \text { conged }\end{array}$ $\begin{array}{lllll}1.755 \pm 0.015 & 1.07 \pm 0.01 & 1.54 \pm 0.07 & 0.895 \pm 0.055 & \text { cottage cheese } \\ 1.92550 .045 & 0.7580 .01 & 1.675 \pm 0.06 & 0.625 \pm 0.01 & \text { ferts }\end{array}$ $\begin{array}{lllll}1.925 \pm 0.045 & 0.75 \pm 0.01 & 1.675 \pm 0.185 & 0.625 \pm 0.005 & \text { fermented goat's } \mathrm{m}\end{array}$ $\begin{array}{lllll}1.975 \pm 0.115 & 1.785 \pm 0.055 & 1.82 \pm 0.06 & 0.77 \pm 0.01 & \text { fermented cabbage }\end{array}$ $\begin{array}{lllll}1.86 \pm 0.06 & 1.70 \pm 0.05 & 2.025 \pm 0.045 & 0.855 \pm 0.105 & \text { fermented cabbage } \\ 2.07 \pm 0.00 & 1.8750 .05 & 1.85 \pm 0.015 & 1.5950 .015 & \text { fermented cabbage }\end{array}$ $\begin{array}{llllll}2.07 \pm 0.00 & 1.875 \pm 0.005 & 1.845 \pm 0.015 & 1.595 \pm 0.015 & \text { fermented cabbage }\end{array}$ $\begin{array}{lllll}1.89 \pm 0.05 & 1.82 \pm 0.11 & 1.46 \pm 0.12 & 0.81 \pm 0.04 & \text { cottage cheese }\end{array}$ $\begin{array}{lllll}1.68 \pm 0.12 & 1.39 \pm 0.05 & 1.82 \pm 0.19 & 1.715 \pm 0.045 & \text { fermented cabbage }\end{array}$ $\left.\begin{array}{lllll}2.015 \pm 0.035 & 1.02 \pm 0.16 & 1.79 \pm 0.03 & 1.85 \pm 0.01 & \text { fermented cabbage }\end{array}\right]$ $\begin{array}{lllll}1.985 \pm 0.015 & 1.29 \pm 0.01 & 1.785 \pm 0.185 & 1.69 \pm 0.03 & \text { fermented cabbage }\end{array}$

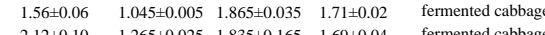
$\begin{array}{lllll}2.12 \pm 0.10 & 1.265 \pm 0.025 & 1.835 \pm 0.165 & 1.69 \pm 0.04 & \text { fermented cabbage } \\ 1.69 \pm 0.075 & 1.375 \pm 0.05 & 1.51 \pm 0.24 & 1.72 \pm 0.03 & \text { fermented cabbage }\end{array}$ $\begin{array}{lllll}1.695 \pm 0.075 & 1.375 \pm 0.05 & 1.51 \pm 0.24 & 1.72 \pm 0.03 & \text { fermented cabbag } \\ 1.970 .07 & 1.175+0.035 & 1.6750 .295 & 1.670 .06 & \text { cottage cheese }\end{array}$ $\begin{array}{lllll}1.97 \pm 0.01 & 1.175 \pm 0.035 & 1.675 \pm 0.295 & 1.67 \pm 0.06 & \text { cottage cheese }\end{array}$ $\begin{array}{lllll}2.05 \pm 0.04 & 1.235 \pm 0.105 & 1.79 \pm 0.01 & 1.61 \pm 0.01 & \text { cottage checse } \\ 1.870 .03 & 1.285 \pm 0.155 & 1.750 .02 & 1.6050 .045 & \text { ryndza }\end{array}$ $\begin{array}{lllll}1.87 \pm 0.03 & 1.285 \pm 0.195 & 1.75 \pm 0.02 & 1.605 \pm 0.045 & \text { bryndza } \\ 1.67051 & 1.015 \pm 0.095 & 1.49 \pm 0.01 & 1.375 \pm 0.035 & \text { cottage }\end{array}$ $\begin{array}{lllll}1.67 \pm 0.51 & 1.015 \pm 0.095 & 1.49 \pm 0.01 & 1.375 \pm 0.035 & \text { cottage cheese }\end{array}$ $\begin{array}{lllll}1.765 \pm 0.095 & 0.631 \pm 0.01 & 1.66 \pm 0.01 & 1.58 \pm 0.10 & \text { cottage cheese }\end{array}$ $\begin{array}{lllll}1.865 \pm 0.035 & 0.145 \pm 0.045 & 1.535 \pm 0.335 & 1.40 \pm 0.01 & \text { soured cream }\end{array}$ $\begin{array}{lllll}2.095 \pm 0.025 & 0.69 \pm 0.00 & 1.37 \pm 0.21 & 1.62 \pm 0.02 & \text { cottage cheese }\end{array}$ $\begin{array}{lllll}2.06 \pm 0.02 & 0.56 \pm 0.07 & 1.655 \pm 0.305 & 1.605 \pm 0.045 & \text { cottage cheese }\end{array}$ $\begin{array}{lllll}1.955 \pm 0.025 & 0.565 \pm 0.005 & 1.49 \pm 0.22 & 1.255 \pm 0.035 & \text { cottage cheese }\end{array}$ $\begin{array}{lllll}1.92 \pm 0.02 & 1.065 \pm 0.065 & 1.47 \pm 0.13 & 1.54 \pm 0.03 & \text { cottage cheese }\end{array}$ $\begin{array}{lllll}2.23 \pm 0.07 & 1.23 \pm 0.04 & 1.528 \pm 0.058 & 1.79 \pm 0.03 & \text { fermented cabbage } \\ 2.17 \pm 0.00 & 0.935 \pm 0.145 & 1.845 \pm 0.125 & 1.575 \pm 0.025 & \text { fermented cabbage }\end{array}$ $\begin{array}{lllll}2.17 \pm 0.00 & 0.935 \pm 0.145 & 1.845 \pm 0.125 & 1.575 \pm 0.025 & \text { fermented cabbage } \\ 2.095 \pm 0.055 & 0.805 \pm 0.025 & 1.695 \pm 0.005 & 1.54 \pm 0.06 & \text { fermented cabbage }\end{array}$ $\begin{array}{lllll}2.095 \pm 0.055 & 0.805 \pm 0.025 & 1.695 \pm 0.005 & 1.54 \pm 0.06 & \text { fermented cabbage } \\ 1.945 \pm 0.025 & 1.015 \pm 0.015 & 1.465 \pm 0.065 & 1.37 \pm 0.04 & \text { fermented cabbage }\end{array}$ $\begin{array}{llllll}1.945 \pm 0.025 & 1.015 \pm 0.015 & 1.465 \pm 0.065 & 1.37 \pm 0.04 & \text { fermented cabbage } \\ 1.97 \pm 0.02 & 1.155 \pm 0.065 & 1.66 \pm 0.04 & 1.52 \pm 0.06 & \text { fermented cabbage }\end{array}$ $\begin{array}{lllll}1.97 \pm 0.02 & 1.155 \pm 0.065 & 1.66 \pm 0.04 & 1.52 \pm 0.06 & \text { fermenteabbage } \\ 2.32+0.03 & 1.06 \pm 0.09 & 1.87 \pm 0.15 & 1.58 \pm 0.11 & \text { fermented cabbage }\end{array}$ $\begin{array}{lllll}2.32 \pm 0.03 & 1.06 \pm 0.09 & 1.87 \pm 0.15 & 1.58 \pm 0.11 & \text { fermed cabbage } \\ 2.045 \pm 0.0055 & 1.29 \pm 0.06 & 1.29 \pm 0.16 & 0.975 \pm 0.055 & \text { fermented cabb }\end{array}$ $\begin{array}{lllll}2.045 \pm 0.0055 & 1.29 \pm 0.06 & 1.29 \pm 16 & 1.5875 .11 & \text { feres } \\ 2.015-0.055 & 1.435 \pm 0.055 & 1.065 \pm 0.165 & 0.97 \pm 0.34 & \text { fermented cabbage }\end{array}$ $\begin{array}{lllll}2.015 \pm 0.055 & 1.435 \pm 0.055 & 1.065 \pm 0.165 & 0.97 \pm 0.34 & \text { formed cabbage } \\ 2.095 \pm 0.015 & 1.83 \pm 0.01 & 1.785 \pm 0.125 & 1.285 \pm 0.045 & \text { fermented cose }\end{array}$ $\begin{array}{lllll}1.665 \pm 0.005 & 1.095 \pm 0.185 & 0.825 \pm 0.005 & 0.685 \pm 0.195 & \text { cottage cheese }\end{array}$ $\begin{array}{lllll}1.895 \pm 0.015 & 0.605 \pm 0.005 & 1.705 \pm 0.285 & 0.99 \pm 0.03 & \text { soured cream }\end{array}$ $\begin{array}{lllll}1.89 \pm 0.13 & 1.17 \pm 0.03 & 2.095 \pm 0.025 & 1.67 \pm 0.00 & \text { soured cream } \\ 2.025+0.05 & 1.325 .0 .015 & 2.21 \pm 0.01 & 1.66550 .155 & \text { sured cream }\end{array}$ $\begin{array}{lllll}2.025 \pm 0.05 & 1.325 \pm 0.015 & 2.21 \pm 0.01 & 1.665 \pm 0.155 & \text { soured cream } \\ 1.950 .07 & 0.935 \pm 0.035 & 1.82 \pm 0.10 & 1.375 \pm 0.015 & \text { cotage cheese }\end{array}$ $\begin{array}{lllll}1.95 \pm 0.07 & 0.935 \pm 0.035 & 1.82 \pm 0.10 & 1.375 \pm 0.015 & \text { cottage cheese }\end{array}$ $\begin{array}{lllll}1.52 \pm 0.00 & 0.555 \pm 0.015 & 1.78 \pm 0.05 & 1.195 \pm 0.005 & \text { bryndza } \\ 1.97 \pm 0.02 & 1.005 \pm 0.025 & 1.895 \pm 0.035 & 1.6050 .025 & \text { ferled }\end{array}$ $\begin{array}{lllll}1.97 \pm 0.02 & 1.005 \pm 0.025 & 1.895 \pm 0.035 & 1.605 \pm 0.025 & \text { fermented cabbage } \\ 2.205 \pm 0.015 & 0.84 \pm 0.15 & 1.975 \pm 0.155 & 1.53 \pm 0.04 & \text { formented cucumb }\end{array}$ $\begin{array}{lllll}2.205 \pm 0.015 & 0.84 \pm 0.15 & 1.975 \pm 0.155 & 1.53 \pm 0.04 & \text { fermented cucum } \\ 1.9250 .025 & 0.780 .16 & 1.66 \pm 0.05 & 1.245 \pm 005 & \text { soured cream }\end{array}$ $\begin{array}{lllll}2.215 \pm 0.025 & 0.78 \pm 0.16 & 1.66 \pm 0.05 & 1.245 \pm 0.005 & \text { soured cream }\end{array}$ $\begin{array}{lllll}1.995 \pm 0.035 & 0.65 \pm 0.09 & 1.455 \pm 0.055 & 1.065 \pm 0.015 & \text { fermented cabbage } \\ 1.4650 .025 & 1.305 \pm 0.025 & 1.940 .02 & 1.5250 .095 & \text { fenmed goat's }\end{array}$ $\begin{array}{lllll}1.465 \pm 0.025 & 1.305 \pm 0.025 & 1.94 \pm 0.02 & 1.525 \pm 0.095 & \text { fermented goat's } \\ 2.325 \pm 0.05 & 1.52 \pm 0.05 & 1.235 .0 .015 & 1.775 \pm 0.045 & \text { fors }\end{array}$ $\left.\begin{array}{lllll}2.215 \pm 0.015 & 1.845 \pm 0.005 & 1.435 \pm 0.115 & 1.82 \pm 0.00 & \text { fermented cabbage }\end{array}\right\} \Delta \mathrm{pHcab} 2.5 \% \mathrm{NaCl}=\Delta \mathrm{pHcuc} 6.0 \% \mathrm{NaCl}$ $\begin{array}{lllll}1.67 \pm 0.06 & 1.295 \pm 0.015 & 1.31 \pm 0.07 & 1.725 \pm 0.055 & \text { fermented cabbage }\end{array}$ $\begin{array}{lllll}2.035 \pm 0.095 & 1.34 \pm 0.04 & 1.26 \pm 0.21 & 1.77 \pm 0.02 & \text { fermented cucumb }\end{array}$ $\begin{array}{lllll}2.155 \pm 0.035 & 0.90 \pm 0.08 & 1.38 \pm 0.04 & 1.785 \pm 0.015 & \text { bryndza } \\ 1.83 \pm 0.04 & 0.68 \pm 0.16 & 0.55 \pm 0.03 & 1.705 \pm 0.025 & \text { fermented cow's } m\end{array}$ $\begin{array}{lllll}1.83 \pm 0.04 & 0.68 \pm 0.16 & 0.85 \pm 0.03 & 1.705 \pm 0.025 & \text { fermented cow's m } \\ 1.515 \pm 0.125 & 1.84 \pm 0.01 & 1.31 \pm 0.15 & 1.715 \pm 0.035 & \text { soured cream }\end{array}$ $\begin{array}{lllll}1.775 \pm 0.015 & 1.62 \pm 0.10 & 1.23 \pm 0.07 & 1.875 \pm 0.355 & \text { fermented cab }\end{array}$ $\begin{array}{lllll}1.805 \pm 0.105 & 1.59 \pm 0.03 & 1.14 \pm 0.03 & 1.355 \pm 0.045 & \text { fermented cabbage } \\ 1.38 \pm 0.0 & 1.65 \pm 0.125 & 1.155 \pm 0.055 & 0.89 \pm 0.04 & \text { cottage cheese }\end{array}$ $\begin{array}{lllll}1.38 \pm 0.20 & 1.695 \pm 0.125 & 1.155 \pm 0.055 & 0.89 \pm 0.04 & \text { cottage cheese }\end{array}$ $\begin{array}{llll}0.545 \pm 0.005 & 1.475 \pm 0.085 & 1.955 \pm 0.005 & 0.71 \pm 0.13\end{array}$ fermented cab

$\Delta \mathrm{pHcab}>\Delta \mathrm{pHcuc}$ II

$\Delta \mathrm{pHcab}>\Delta \mathrm{pHcab} 2.5 \% \mathrm{NaCl}$

$-\Delta$ pHcuc $=\Delta$ pHcuc $6.0 \% \mathrm{NaCl}$

$\Delta$ pHcab2.5\% NaCl$=\Delta$ pHcuc $6.0 \% \mathrm{NaCl}$

$\Delta \mathrm{pHcab}=\Delta \mathrm{pHcu}$

$\Delta \mathrm{pHcab}=\Delta \mathrm{pHcab} 2.5 \% \mathrm{NaCl}$

$\Delta \mathrm{pHcuc}=\Delta \mathrm{pHcuc} 6.0 \% \mathrm{NaCl}$

$\Delta \mathrm{pHcab} 2.5 \% \mathrm{NaCl}=\Delta \mathrm{pHcuc} 6.0 \% \mathrm{NaCl}$

$\mathrm{pHcab}>\Delta \mathrm{pHcuc}$

$\Delta \mathrm{pHcab} 2.5 \% \mathrm{NaCl}>\Delta \mathrm{pHcuc} 6.0 \% \mathrm{NaCl}$

$\Delta \mathrm{pHcab}>\Delta \mathrm{pHcuc}$

$\Delta \mathrm{pHcab} 2.5 \% \mathrm{NaCl}=\Delta \mathrm{pHcuc} 6.0 \% \mathrm{NaCl}$

$\Delta \mathrm{pHcab}=\Delta \mathrm{pHcuc}$

IV

$\Delta \mathrm{pHcab}>\Delta \mathrm{pH}$ $\Delta$ pHcuc $>\Delta$ pHcuc $6.0 \% \mathrm{NaCl}$

$\Delta \mathrm{pHcab}=\Delta \mathrm{pHcuc}$

$\Delta \mathrm{pHcab} 2.5 \% \mathrm{NaCl}=\Delta \mathrm{pHcuc} 6.0 \% \mathrm{NaCl}$

$\Delta \mathrm{pHcab}=\Delta \mathrm{pHcuc}$

$\Delta \mathrm{pHcab} 2.5 \% \mathrm{NaCl}<\Delta \mathrm{pHcuc} 6.0 \% \mathrm{NaCl}$

VI

$\Delta \mathrm{pHcab}>\Delta \mathrm{pHcab} 2.5 \% \mathrm{NaCl}$

$-\Delta \mathrm{pHcuc}=\Delta \mathrm{pHcuc} 6.0 \% \mathrm{NaCl}$

$\Delta \mathrm{pHcab}>\Delta \mathrm{pHcuc}$

$\Delta \mathrm{pHcab} 2.5 \% \mathrm{NaCl}<\Delta \mathrm{pHcuc} 6.0 \% \mathrm{NaCl}$

$\Delta \mathrm{pHcab}>\Delta \mathrm{pHcuc}$
$\Delta \mathrm{pHcab} 2.5 \% \mathrm{NaCl}>\Delta \mathrm{pHcuc} 6.0 \% \mathrm{NaCl}$

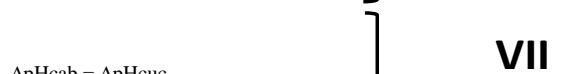

$\Delta \mathrm{pHcab}=\Delta \mathrm{pHcuc}$

$\Delta \mathrm{pHcuc}>\Delta \mathrm{pHcuc} 6.0 \% \mathrm{NaCl}$

$\Delta \mathrm{pHcab}>\Delta \mathrm{pHcuc}$

V

$\Delta \mathrm{pHcab}=\Delta \mathrm{pHcab} 2.5 \% \mathrm{NaCl}$ $\Delta \mathrm{pH}_{\text {cuc }}>\Delta \mathrm{pH}$ cuc $6.0 \% \mathrm{NaCl}$ $\Delta \mathrm{pH}$ pHcuc $<\Delta \mathrm{pHcuc} 6.0 \% \mathrm{NaCl}$

$\Delta \mathrm{pHcab}=\Delta \mathrm{pHcab} 2.5 \% \mathrm{NaCl}$

$\Delta \mathrm{pHcuc}<\Delta$ pHcuc $6.0 \% \mathrm{NaC}$

$\Delta \mathrm{pHcab} 2.5 \% \mathrm{NaCl}=\Delta \mathrm{pHcuc} 6.0 \% \mathrm{NaC}$

$\Delta \mathrm{pHcab}>\Delta \mathrm{pHcuc}$ 
(a)

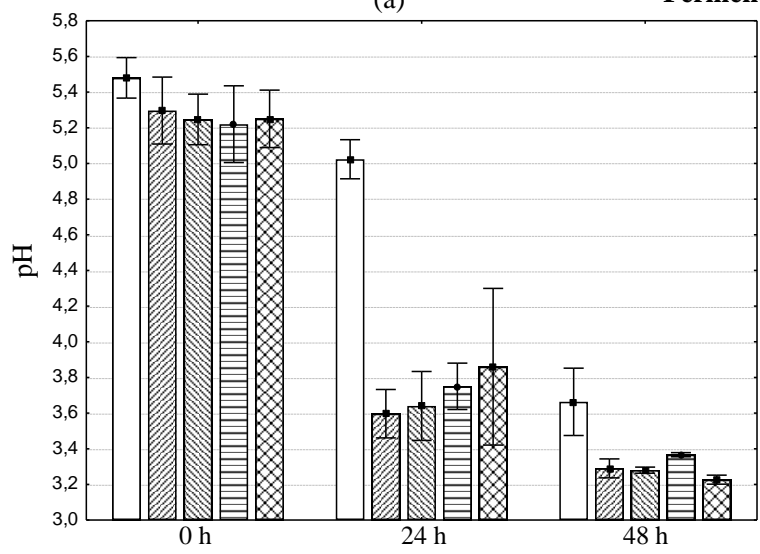

time of fermentation

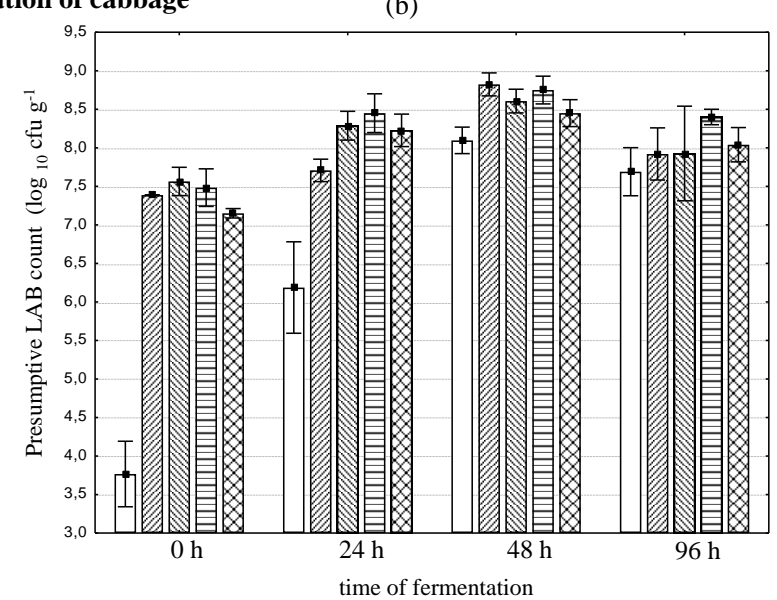

(d)
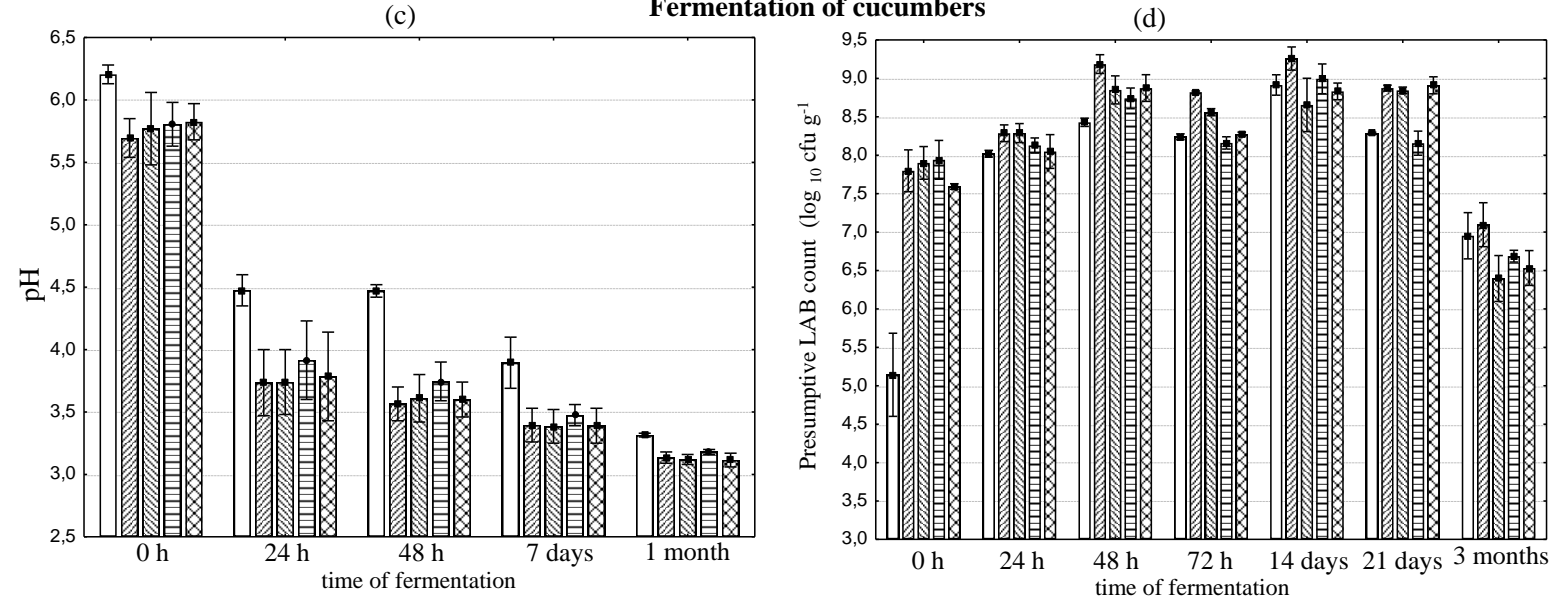

$\square \begin{array}{ll}\text { Spontaneous } & \text { L. plantarum-inoculated } \\ \text { fermentation (control); } & \text { vegetables: }\end{array}$

Figure 2 Changes in $\mathrm{pH}$ and presumptive lactic acid bacteria (LAB) counts during fermentation of vegetables

Four L. plantarum strains, selected for vegetable fermentation, had a different significant difference between the vegetables inoculated by different $L$. $\Delta \mathrm{pH}$ values on pasteurized vegetable-based media. But there was no statistically

plantarum strains. (Table 2)

Table 2 The $\Delta \mathrm{pH}$ values on vegetable-based media and vegetables inoculated by $L$. plantarum srains

\begin{tabular}{|c|c|c|c|c|c|c|c|c|}
\hline \multirow{3}{*}{ Strains } & \multicolumn{8}{|c|}{ Media/vegetables, time of fermentation, $\Delta \mathrm{pH}$} \\
\hline & \multicolumn{2}{|c|}{ cabbage-based medium, $8 \mathrm{~h}$} & \multicolumn{2}{|c|}{ cabbage $+2.5 \% \mathrm{NaCl}$} & \multicolumn{2}{|c|}{ cucumber-based medium, $8 \mathrm{~h}$} & \multicolumn{2}{|c|}{ cucumbers $+6 \% \mathrm{NaCl}$} \\
\hline & pasteurized & $\begin{array}{c}\text { pasteurized }+ \\
2.5 \% \mathrm{NaCl}\end{array}$ & $24 \mathrm{~h}$ & $48 \mathrm{~h}$ & pasteurized & $\begin{array}{c}\text { pasteurized + } \\
6.0 \% \mathrm{NaCl}\end{array}$ & $24 \mathrm{~h}$ & $48 \mathrm{~h}$ \\
\hline $\begin{array}{l}\text { Control } \\
\text { (non-inoculated) }\end{array}$ & $0.03 \pm 0.02$ & $0.05 \pm 0.01$ & $0.45 \pm 0.07$ & $1.81 \pm 0.08$ & $0.06 \pm 0.05$ & $0.04 \pm 0.04$ & $1.73 \pm 0.28$ & $1.73 \pm 0.03$ \\
\hline L. plantarum 47SM & $2.21 \pm 0.03$ & $0.78 \pm 0.22$ & $1.70 \pm 0.05$ & $2.00 \pm 0.13$ & $1.66 \pm 0.07$ & $1.24 \pm 0.01$ & $1.96 \pm 0.15$ & $2.13 \pm 0.02$ \\
\hline L. plantarum $691 \mathrm{~T}$ & $2.05 \pm 0.05$ & $1.23 \pm 0.14$ & $1.60 \pm 0.29$ & $1.96 \pm 0.14$ & $1.79 \pm 0.01$ & $1.61 \pm 0.01$ & $2.03 \pm 0.04$ & $2.16 \pm 0.14$ \\
\hline L. plantarum $1047 \mathrm{~K}$ & $1.94 \pm 0.08$ & $1.35 \pm 0.30$ & $1.47 \pm 0.13$ & $1.84 \pm 0.18$ & $1.90 \pm 0.24$ & $1.43 \pm 0.03$ & $1.89 \pm 0.19$ & $2.00 \pm 0.02$ \\
\hline L. plantarum $952 \mathrm{~K}$ & $1.68 \pm 0.23$ & $1.85 \pm 0.06$ & $1.39 \pm 0.29$ & $2.02 \pm 0.13$ & $1.35 \pm 0.16$ & $1.11 \pm 0.01$ & $2.04 \pm 0.29$ & $2.22 \pm 0.01$ \\
\hline
\end{tabular}

Multivariate analysis for the correlation between values of $\Delta \mathrm{pH}$ was performed of the four $L$. plantarum strains depending on vegetable-based media and vegetables (Table 3). There were significant strong positive correlations between values of $\Delta \mathrm{pH}$, that were found on vegetable-based media and values of $\Delta \mathrm{pH}$ on fermented vegetables.

The selection of LAB as suitable starters for production of fermented vegetables is a multi-stage process. The most common approach is the use of sterilized vegetable-based media, mainly vegetable juices that can be stored at $-20{ }^{\circ} \mathrm{C}$ before use (Gardner et al., 2001; Di Cagno et al., 2008). The kinetics of growth and acidification were determined by authors after incubation during $24 \mathrm{~h}$ (Di Cagno et al., 2008) or up to $60 \mathrm{~h}$ (Gardner et al., 2001). As was shown by Di Cagno et al. (2008), acidification for the lactic acid bacteria strains has reached values of $\Delta \mathrm{pH}$ which ranged from 2.32 to 3.05 after $24 \mathrm{~h}$ of incubation in vegetable juice media. In the present study, after $8 \mathrm{~h}$ of incubation, the $\mathrm{pH}$ values of inoculated vegetable-based media decreased by $0.125-2.33$ units from initial value, whereas in control media - only on 0.03-0.06 units. The decrease of $\mathrm{pH}$ reflects the growth of lactic acid bacteria and these results indicate that the $L$. plantarum strains studied were able to proliferate in the vegetables-based media used. So, the screening approach used in this work is simple and less time consuming as compared to other screening methods.

The most studies on $\mathrm{LAB}$ resistance to $\mathrm{NaCl}$ have been performed using MRS medium, the standard rich medium used for laboratory cultivations (Bevilacqua et al., 2010). But the cultivation of L. plantarum on MRS has to be regarded as significantly different from the conditions encountered by L. plantarum during vegetable fermentations, as was shown by authors (Di Cagno et al., 2013). As was shown by Filannino et al. (2016), the most prominent phenotypic dissimilarities observed in cells grown on carrot and pineapple were related to carbon and nitrogen metabolism, respectively. It's well known that the bacteria response to hyperosmolarity is related to the ability of cells to accumulate compatible solutes that include amino acids, amino acid derivatives, polyols, and sugars (Csonka, 1989). To our knowledge, no papers in which the effect of different concentration of $\mathrm{NaCl}$ on growth L. plantarum strains in several vegetable-based media has been compared were published, and this makes it very difficult to compare results of our work with those obtained by others authors Further studies are required to determine the mechanisms responsible for the $\mathrm{NaCl}$ resistance during growth in vegetables. 
Table 3 Correlations between $\Delta \mathrm{pH}$ values on vegetable-based media and vegetables inoculated by $L$. plantarum srains

\begin{tabular}{|c|c|c|c|c|c|c|c|}
\hline \multirow{3}{*}{$\begin{array}{l}\text { Media/vegetables, } \\
\text { time of fermentation }\end{array}$} & \multicolumn{3}{|c|}{ Vegetables-based media, $8 \mathrm{~h}$ incubation } & \multicolumn{4}{|c|}{ Fermented vegetables } \\
\hline & $\begin{array}{c}\text { cabbage-based } \\
\text { media }\end{array}$ & $\begin{array}{r}\text { cucumb } \\
\mathrm{m}\end{array}$ & based & $\begin{array}{r}\mathrm{ca} \\
(2.5 \mathrm{c} \\
\end{array}$ & & & \\
\hline & $\begin{array}{l}\text { without } \\
\mathrm{NaCl}\end{array}$ & $\begin{array}{l}\text { without } \\
\mathrm{NaCl}\end{array}$ & $\begin{array}{l}6.0 \% \\
\mathrm{NaCl}\end{array}$ & $24 \mathrm{~h}$ & $48 \mathrm{~h}$ & $24 \mathrm{~h}$ & $48 \mathrm{~h}$ \\
\hline
\end{tabular}

\begin{tabular}{|c|c|c|c|c|c|c|c|c|}
\hline $\begin{array}{l}\text { Vegetables-based media, } 8 \text { h } \\
\text { cabbage-based }\end{array}$ & 1.0 & & & & & & & \\
\hline cabbage-based+2.5\% NaCl & 0.67 & 1.00 & & & & & & \\
\hline cucumber-based & $0.96 *$ & 0.69 & 1.00 & & & & & \\
\hline cucumber-based +6.0\% NaCl & $0.95^{*}$ & 0.73 & $0.98 *$ & 1.00 & & & & \\
\hline $\begin{array}{l}\text { Fermented vegetables } \\
\text { cabbage }(2.5 \% \mathrm{NaCl}), 24 \mathrm{~h}\end{array}$ & $1.00^{*}$ & 0.67 & $0.95 *$ & $0.95 *$ & 1.00 & & & \\
\hline cabbage $(2.5 \% \mathrm{NaCl}), 48 \mathrm{~h}$ & 0.64 & 0.56 & 0.44 & 0.51 & 0.68 & 1.00 & & \\
\hline cucumbers $(6.0 \% \mathrm{NaCl}), 24 \mathrm{~h}$ & 0.82 & 0.83 & 0.73 & 0.81 & 0.84 & 0.87 & 1.00 & \\
\hline Cucumbers $(6.0 \% \mathrm{NaCl}), 48 \mathrm{~h}$ & $0.88^{*}$ & 0.88 & 0.82 & 0.86 & $0.90 *$ & 0.83 & $0.98 *$ & 1.00 \\
\hline
\end{tabular}

Dynamics of $\mathrm{pH}$ decrease and presumptive LAB count increase during fermentation of inoculated cabbage were different from those of cucumbers. This may be due to a smaller number of the indigenous presumptive LAB in raw cabbage than in cucumbers $\left(3.76 \pm 0.73 \log _{10} \mathrm{cfu} \mathrm{g}^{-1}\right.$ and $5.14 \pm 1.08 \log _{10} \mathrm{cfu} \mathrm{g}^{-1}$ respectively). And although, $L$. plantarum strains addition in high numbers ensured a prevalence of these strains in the inoculated vegetables, the inoculated L. plantarum strains competed not only with indigenous $\mathrm{LAB}$, but with all microorganisms of the raw vegetables. Many authors have reported that the use of lactic acid bacteria strains as starters in the fermentation of vegetables significantly increased acid production, when compared with spontaneously fermented vegetables (Desai and Sheth, 1997; Joyce et al., 2018).

Difference in lactic acid production rate by $L$. plantarum strains during growth on vegetable-based media and vegetables fermentation may be due to the fact that vegetable-based media were pasteurized, in contrast to raw vegetables with native microflora. Another reason may be the difference in the nutritional composition of cabbage and cucumbers and their availability. So, for the preparation of vegetable-based media the vegetables were homogenized. Before fermentation, the cabbage was shredded, while cucumbers were fermented whole. The significant strong positive correlations between $\Delta \mathrm{pH}$ values on cabbage- and cucumber-based media and significant strong positive correlations between $\Delta \mathrm{pH}$ values on vegetable-based media and fermented cabbage after $24 \mathrm{~h}$ incubation may also indicate an increase in the availability of nutrients. At the same time, significant correlations between cucumber-based media and fermented cucumbers were not detected.

\section{CONCLUSION}

In conclusion, to our knowledge, this is a first study in which a simple and fas approach was used to evaluate the potential of L. plantarum strains as starters for vegetable fermentation. Stimulating effect of $\mathrm{NaCl}$ in vegetable-based medium on the acidification, during exponential growth phase of $L$. plantarum strains has been found for the first time. The four L. plantarum strains selected through this research are promising starter cultures, as was shown in the laboratory-scale fermentation of vegetables. The impact of these strains on the organoleptic properties of the fermented vegetables will be the object of further investigation. Strong positive correlations were found between values of $\Delta \mathrm{pH}$ on vegetablebased media (without $\mathrm{NaCl}$ ) and values of $\Delta \mathrm{pH}$ that were found during vegetable fermentations, which suggests that the screening process in vegetable-based media after $8 \mathrm{~h}$ incubation could be effective.

\section{REFERENCES}

BEHERA, S. S., RAY, R. C., ZDOLEC, N. 2018. Lactobacillus plantarum with functional properties: an approach to increase safety and self-life of fermented foods. BioMed Reseach International, 2018 https://doi.org/10.1155/2018/9361614

BEVILACQUA, A., ALTIERI, C., CORBO, M. R., SINIGAGLIA, M., OUOBA L. I. I. 2010. Characterization of lactic acid bacteria isolated from Italian Bella di Cerignola table olives: selection of potential multifunctional starter cultures Journal of Food Science, 75, M536-544. https://doi.org/10.1111/j.17503841.2010.01793.x

COGAN, T. M., BARBOSA, M., BEUVIER, E., BIANCHI-SALVADORE, B., COCCONCELLI, P. H., FERNANDEZ, P. S., GOMEZ I., KALANTZOUPOULOS G., LEDDA A., MEDINA M., REA M. C., RODRIGUEZ E. 1997. Characterization of the lactic acid bacteria in artisanal dairy products. Journal of Dairy Research, 64, 409-421.

CSONKA, L. N. 1989. Physiological and genetic responses of bacteria to osmotic stress. Microbiological Reviews, 53, 121-147.

DESAI, P., SHETH, T. 1997. Controlled fermentation of vegetables using mixed inoculum of lactic cultures. Journal of Food Science and Technology, 34, 155158.
DAS, R., PANDEY, H., DAS, B., SARKAR S. 2016. Fermentation and its application in vegetable preservation: a review. International Journal of Fermentation Technology, 6, 207-217. https://doi.org/10.5958/22779396.2016.00044.1

DI CAGNO, R., SURICO, R. F., SIRAGUSA, S., DE ANGELIS, M. PARADISO, A., MINERVINI, F., DE GARA, L., GOBBETTI, M. 2008. Selection and use of autochthonous mixed starter for lactic acid fermentation of carrots, French beans or marrows. International Journal of Food Microbiology, 127, 220-228. https://doi.org/10.1016/j.ijfoodmicro.2008.07.010

DI CAGNO, R., CODA, R., DE ANGELIS, M., GOBBETTI, M. 2013 Exploitation of vegetables and fruits through lactic acid fermentation. Food Microbiology, 33, 1-10. https://doi.org/10.1016/j.fm.2012.09.003

FILANNINO, P., DI CAGNO, R., CRECCHIO, C., DE VIRGILIO, C., DE ANGELIS, M. \& GOBBETTI, M. 2016. Transcriptional reprogramming and phenotypic switching associated with the adaptation of Lactobacillus plantarum C2 to plant niches. Scientific Reports, 6, 27392 https://doi.org/10.1038/srep27392

GARDNER, N. J., SAVARD, T., OBERMEIER, P., CALDWELL, G., CHAMPAGNE, C. P. 2001. Selection and characterization of mixed starter cultures for lactic acid fermentation of carrot, cabbage, beet and onion vegetable mixtures. International Journal of Food Microbiology, 64, 261-275.

GORNY, J. 2006. Microbial contamination of fresh fruits and vegetables. In Microbiology of Fruits and Vegetables ed. Sapers, G. M., Gorny, J. R. and Yousef, A. E. Y. pp. 3-32. Boca Raton: CRC Press.

HARRIS, L. 1998. The microbiology of vegetable fermentations. In Microbiology of Fermented Foods 2nd ed. ed Wood, B. J. B. pp. 46-72. London: Blackie Academic and Professional.

JOYCE, O. T., CHAKRABORTY, S. K., TRIPATHI, M. K., KOTWALIWALE, N., CHANDRA, P. 2018. Quality characteristics of sauerkraut fermented by using a Lactobacillus paracasei starter culture grown in tofu whey. Food Science Technology, 24, 187-197. https://doi.org/10.1177/1082013217741798

KAROVICOOVÁ, J., KOHAJDOVÁ, Z. 2003. Lactic acid fermented vegetable juices. Horticultural Science, 30, 152-158. https://doi.org/10.17221/3878 HORTSCI

KIM, M., CHUN, J. 2005. Bacterial community structure in kimchi, a Korean fermented vegetable food, as revealed by $16 \mathrm{~S}$ rRNA gene analysis. International Journal of Food Microbiology, 103, 91-96. https://doi.org/10.1016/j.ijfoodmicro.2004.11.030

LIVINSKA, O., IVASCHENKO, O., GARMASHEVA, I., KOVALENKO, N. 2016. The screening of lactic acid bacteria with antioxidant properties. AIMS Microbiology 2, 447-459. https://doi.org/10.3934/microbiol.2016.4.447

LEE, K. W., SHIM, J. M., YAO, Z., KIM, J. A., KIM. J. H. 2018. Properties of Kimchi fermented with GABA-producing lactic acid bacteria as a starter. Journal of Microbiology and Biotechnology, 28, 534-541. https://doi.org/10.4041/jmb.1709.09011

MONTET, D., LOISEAU, G., ZAKHIA-ROZIS, N. 2006. Microbial technology of fermented vegetables. In Microbial Biotechnology in Horticulture ed Ray, R. C.,Ward, O.P. pp. 309-343. Enfield, NH: Science Publishers Inc.

REZAK, S., KOK, C. R., HEERMANN, M., HUTKINS R. 2018. Fermented foods as a dietary source of live organisms. Frontiers of Microbiology, 9, 1785. https://doi.org/10.3389/fmicb.2018.01785

ROUSHDY, I. M. 1999. Molecular and phenotypic characterization of lactic acid bacteria isolated from laban rayeb. Annals of Agricultural Science Cairo, 44 617-630.

SOLTAN DALLAL, M. M., ZAMANIAHARI, S., DAVOODABADI A., HOSSEINI, M., RAJABI, Z. 2017. Identification and characterization of probiotic lactic acid bacteria isolated from traditional Persian pickled vegetables. GMS Hygiene and Infection Control, 12. https://doi.org/10.3205/dgkh000300

TAMANG, J. P., TAMANG, B., SCHILLINGER, U., GUIGAS, C. HOLZAPFEL, W. H. 2009. Functional properties of lactic acid bacteria isolated from ethnic fermented vegetables of the Himalayas. International Journal of 
Food Microbiology, $\quad 135, \quad 28-33$.

https://doi.org/10.1016/j.ijfoodmicro.2009.07.016

TAMMINEN, M., JOUTSJOKI, T., SJÖBLOM, M., JOUTSEN, M., PALVA,

A., RYHÄNEN, E.-L. 2004. Screening of lactic acid bacteria from fermented vegetables by carbohydrate profiling and PCR-ELISA. Letters of Applied Microbiology, 39, 439-444. https://doi.org/10.1111/j.1472-765X.2004.01607.x

VASYLIUK, O. M., KOVALENKO, N. K., HARMASHEVA, I. L. \&

OLESHCHENKO, L. T. 2014. Isolation and identification of bacteria of Lactobacillus genus from fermented products in diferent regions of Ukraine. Mikrobiologichny Zhurnal, 76, 2-9.

VASYLIUK, O. M., KOVALENKO, N. K., HARMASHEVA, I. L. 2014a Antagonistic properties of Lactobacillus plantarum strains, isolated from traditional fermented products of Ukraine. Mikrobiologichny Zhurnal, 76, 24-30.

VASULYK, O. M., GARMASHEVA, I. L., KOVALENKO, N. K. 2014b. Probiotic properties of strains of Lactobacillus plantarum isolated from fermented products. Microbiology \& Biotechnology, 27, 23-31.

VIANDER B., MAKI M., PALVA A. 2003. Impact of low salt concentration, salt quality on natural large-scale sauerkraut fermentation. Food Microbiology 20, 391-395. https://doi.org/10.1016/S0740-0020(02)00150-8 